\title{
CHEMOSPHERE
}

\section{Characteristics of organic precursors and their relationship with disinfection by-products}

\author{
E.E. Chang ${ }^{\mathrm{a}, *}$, Pen-Chi Chiang ${ }^{\mathrm{b}}$, Ya-Wen Ko ${ }^{\mathrm{c}}$, Wen-Hsieh Lan ${ }^{\mathrm{b}}$ \\ a Department of Biochemistry, Taipei Medical University, Taipei, Taiwan, ROC \\ ${ }^{\mathrm{b}}$ Graduate Institute of Environmental Engineering, National Taiwan University, Taipei, Taiwan, ROC \\ ${ }^{\mathrm{c}}$ Department of Environmental Engineering, Da-Yeh University, Taipei, Taiwan, ROC
}

Received 7 February 2000; received in revised form 17 May 2000; accepted 17 May 2000

\begin{abstract}
The molecular weight distribution and chemical composition of precursors and their relationship with disinfection by-products (DBPs) were investigated. Most of the organic matter responsible for the major DBP precursors in the PanHsin water are small compounds with a molecular weight less than $1 \mathrm{kDa}$. The hydrophobic acids display the greatest ability to produce DBP. Therefore, effective removal of small molecules or hydrophobic acidic organics prior to disinfection process will significantly reduce the DBP concentration in the finished water. Although the coagulation process is effective in removing large organic precursors and the removal efficiencies of $\mathrm{CHCl}_{3}$ formation potential and organic carbon increase proportionally to the molecular weight of the precursors, the conventional treatment methods have limited efficiency in eliminating small precursors, which have high DBP formation potential. (c) 2001 Elsevier Science Ltd. All rights reserved.
\end{abstract}

Keywords: Disinfection by-product; Hydrophilic; Hydrophobic; Molecular weight; Precursor

\section{Introduction}

It is necessary to understand the characteristics of source water, including the molecular weight of organics, chlorine demand, DBPFP (disinfection by-product formation potential), UV absorbance, functional groups, acidity, and alkalinity (Owen et al., 1993), for selecting the effective water purification processes. Since the molecular weight of precursors affects the treatability of DOM (dissolved organic matter) and DBP, the determination of molecular weight distribution of organic precursors in source water will assist in the enhancement of removal efficiency in water treatment processes.

\footnotetext{
${ }^{*}$ Corresponding author. Tel.: +886-22736-9236; fax: +88622736-9236.

E-mail address: eechang@tmc.edu.tw (E.E. Chang).
}

The previous researchers (Lou and Chiang, 1991) used a colloidal layered column to analyze the distribution pattern of molecular weight of precursors in surface water of Taiwan and found that the molecular weight ranged from $200 \mathrm{Da}$ to $50 \mathrm{kDa}$. Other researchers (Chang, 1999) utilized high performance liquid chromatograph (HPLC) to analyze the molecular weight distribution of DOM in four major rivers in Taiwan. The result revealed that $64-87 \%$ of the precursors have molecular weight in the range 1-30 kDa. The group of small precursors (molecular weight less than $1 \mathrm{kDa}$ ) accounted for 9-26\%. Veenstra and Schnoor (1980) analyzed the organics from the Iowa River using Sephadex G-75, and reported that $87 \%$ of the molecular weight of the TTHM (total trihalomethane) precursors was less than $3 \mathrm{kDa}$. Huang and Yeh (1997) studied the water samples from each treatment process of FengShan Waterworks (Taiwan) and found that the removal of NPDOC (non-purgeable dissolved organic carbon) 
with the molecular weight $>1.7 \mathrm{kDa}$ was the highest $(57 \%)$, and that the removal efficiency of DOM increased with molecular weight. Collins et al. (1986) investigated the removal efficiency of TOC (total organic carbon) in raw water samples from rivers, reservoirs and groundwater by the conventional treatment process. They reported that the removal efficiency of TOC for small molecules (molecular weight $<500 \mathrm{Da}$ ) by coagulation process was insignificant and the maximum TOC removal efficiency was observed at the molecular weight ranging from 1 to $10 \mathrm{kDa}$.

The purposes of this research were to investigate the molecular weight distribution and the chemical composition of organic matter and their relationship with the DBP formation. The water samples prepared in this study included commercial humic acids, as well as source water samples taken from Pan-Hsin Waterworks. Furthermore, the molecular weight distribution patterns of DOC and DBPFP in the effluents of each unit process were analyzed to determine the efficiency of treatment process in removing DBP precursors.

\section{Material and methods}

\subsection{Sample preparations}

Three samples were used in this study: (1) raw water from Chang-Hsin Waterworks with the addition of a commercial humic acid (Aldrich), Aldrich-HA, (2) raw water from Chang-Hsin Waterworks with the addition of a commercial humic acid (Tokyo Chemical), TCIHA, and (3) raw water samples from Pan-Hsin Waterworks (taken in March 1998). Prior to the membrane separation, samples were treated with glass-fiber filter media (Whatman GF/C) to prevent the membrane from clogging.

\subsection{Membrane separation}

Ultrafiltration membranes (A/G Technology) were used to separate the samples into five subgroups according to the molecular weight: $<1,1-5,5-10,10-30$, and $>30 \mathrm{kDa}$. The reservation solution $(0.1 \mathrm{M} \mathrm{NaOH})$ inside the membrane system was first withdrawn then rinsed the system with de-ionized water (Milli-Q). Pumps (MP-3, Rikakikai) were then turned on to feed the sample continuously into the membrane system. When the pressure arrived at $69 \mathrm{kPa}$, the filtrate was collected until the expected volume was reached.

After the operation, the membrane system was rinsed with de-ionized water and the freshly prepared $0.5 \%$ Terg-A-Zyme solution and the $0.5 \mathrm{M} \mathrm{NaOH}$ solution were used consecutively to remove the residual contaminants. Finally, a $0.1 \mathrm{M} \mathrm{NaOH}$ solution was introduced into the membrane system as a reservation agent.

\subsection{XAD-8 extraction}

The organic matter in the Pan-Hsin raw water was extracted with an XAD-8 resin and separated into four fractions: hydrophilic fraction, hydrophobic bases, hydrophobic neutrals, and hydrophobic acids. Two glass columns $(3.5-\mathrm{cm}$ diameter, $50-\mathrm{cm}$ long) were packed with pre-conditioned Amberlite XAD-8 resin; the extraction resin was cleaned and prepared according to the procedures described in Standard Methods (APHA, 1995).

Raw water was first adjusted to $\mathrm{pH} 7$ using $\mathrm{HCl}$ and $\mathrm{NaOH}$ solutions, and drawn to pass through the $20-\mu \mathrm{m}$, $1-\mu \mathrm{m}$, and $0.45-\mu \mathrm{m}$ filter, sequentially. The water sample was then fed at a rate of $150 \mathrm{ml} / \mathrm{min}$ into the first column (column 1). The effluent from column 1 was adjusted to $\mathrm{pH} 2$ using $\mathrm{HCl}$ and then pumped into the second resin column (column 2) to collecthydrophilic organic material. $\mathrm{HCl}(0.1 \mathrm{M})$ was used to back-elute the column 1 to wash out the hydrophobic base fraction. $\mathrm{NaOH}(0.1 \mathrm{M})$ was used to back-elute the column 2 to wash out the hydrophobic acid fraction. Resin was then unpacked, dried at $60^{\circ} \mathrm{C}$ for $72 \mathrm{~h}$, and extracted in a Soxhlet extractor with methanol for $12 \mathrm{~h}$ and the extract represents the hydrophobic neutral fraction.

\subsection{Analytical methods}

Two major groups of chlorinated DBP, including THM and HAA (haloacetic acid), were analyzed. The formation potential experiments for DBPs were conducted with a 7-day incubation period following the introduction of the $\mathrm{NaOCl}$ solution and phosphate buffer ( $\mathrm{pH}$ 7.0). The applied chlorine concentration was about $45 \mathrm{mg} / \mathrm{l}$, which was determined from the preliminary studies and would provide a free residual chlorine of at least $3 \mathrm{mg} / \mathrm{l}$ at the end of the incubation period. The analysis of residual chlorine was performed using the DPD (N,N-diethyl- $p$-phenylene-diamine) ferrous titration method. All the detailed analyses followed the QA/ QC programs set forth in Standard Methods (APHA, 1995). The DOC (O.I.C. Model 700) analysis was performed following the UV-persulfate technique using the infrared carbon dioxide analyzer and calibrated with the potassium hydrogen phthalate standard.

THM was extracted with $n$-pentane according to Standard Methods (method 6232B, APHA, 1995). The extract was then analyzed using a GC with a fused silica capillary column (DB-5, $30 \mathrm{~m} \times 0.25 \mathrm{~mm}$ ID, 1.0 $\mu \mathrm{m}$ film thickness) and an electron capture detector. A microextraction procedure (extracting with methyl tertbutyl ether, esterifying with diazomethane) was used to analyze HAA (method 6251B, APHA, 1995). The esterified extract was analyzed using the same GC setup. 


\section{Results and discussion}

\subsection{Molecular weight distribution of DBP precursors in source water}

The molecular weight distribution of DBP precursors, the UV absorbance, THMFP, and HAAFP, in three water samples were first examined. The specific absorbance value, i.e., $\mathrm{UV}_{254}$ /DOC, at various molecular weight ranges for Aldrich-HA, TCI-HA and PanHsin raw water is shown in Fig. 1. It is noted that the amount of conjugated double bond per unit DOC in those three samples follows the order: TCI-HA $>$ Aldrich-HA > Pan-Hsin raw water. High $\mathrm{UV}_{254} / \mathrm{DOC}$ values were observed at a lower molecular weight range. This may imply that at a low molecular weight, there are more conjugated double bonds available to react with chlorine to form DBPs. This speculation was later confirmed by analyzing the amount of DBPs in the water sample and found that a greater DBP formation potential was measured at the low molecular weight.

Fig. 2 shows the DBPFPs per unit DOC for three water samples. Three DBP species, including chloroform, DCAA (dichloroacetic acid), and TCAA (trichloroacetic acid) are the major compounds detected. It is also observed that most of the DBPFPs measured in the TCI-HA sample are distributed in the low molecular weight range $(<10 \mathrm{kDa})$. Similar distribution patterns are found in the Pan-Hsin raw water and Aldrich-HA samples. The fact that organics with small molecular weight play an important role in forming DBPs indicates the importance of their effective removal prior to disinfection process for reducing DBP concentrations in the finished water.

Fig. 3 presents the relationship between the total cumulated DBPFP/DOC (the sum of DBPs measured) and the molecular weight. The trend of three curves shown in Fig. 3 indicates that the DBPFP/DOC values increase slightly as the molecular weight greater than

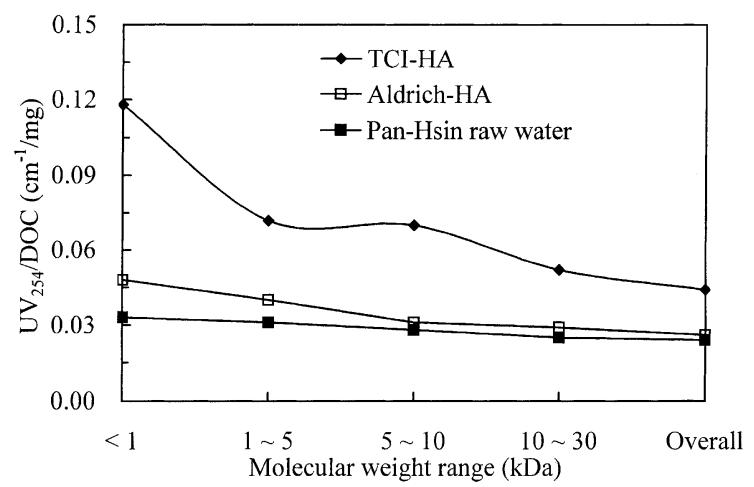

Fig. 1. The molecular weight distribution of three samples and their corresponding $\mathrm{UV}_{254} / \mathrm{DOC}$ values.
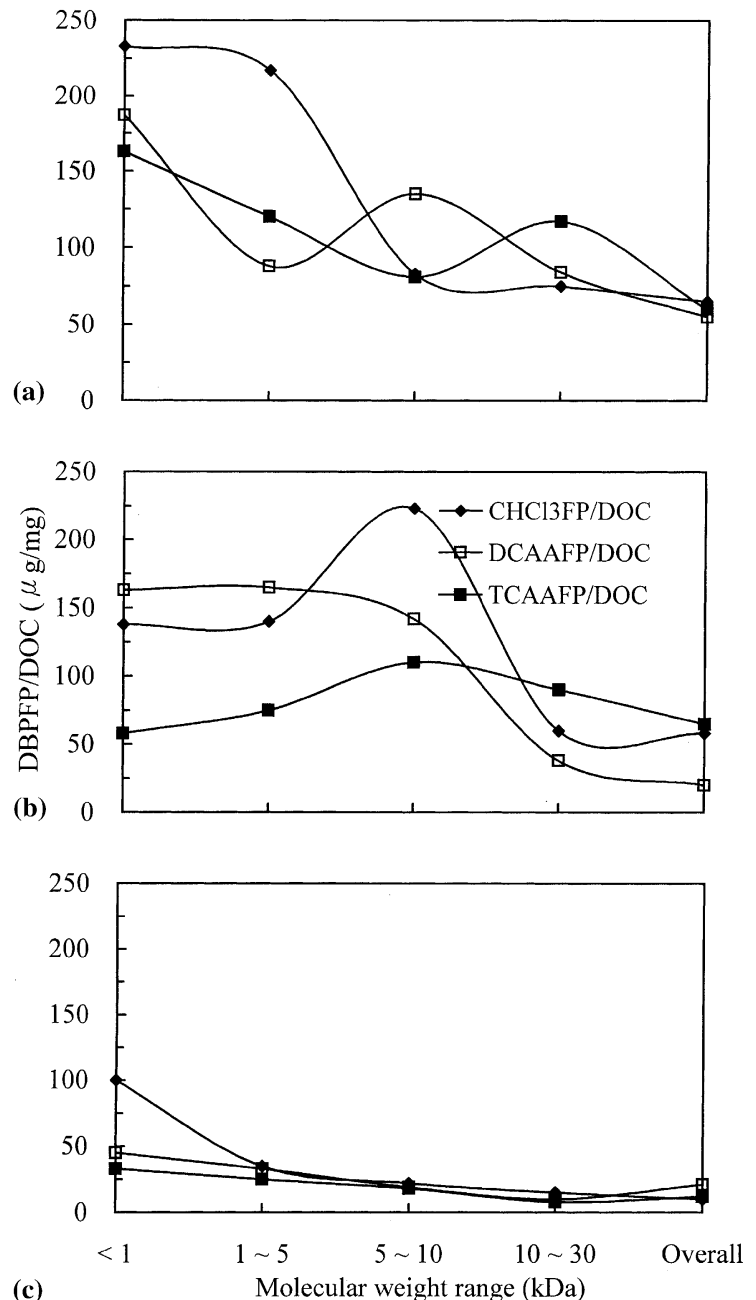

Fig. 2. The relationship between the molecular weight distribution and the corresponding DBPFP/DOC formation for three waters. (a) TCI-HA; (b) Aldrich-HA; (c) Pan Hsin raw water. The symbols used in (b) apply to others.

$10 \mathrm{kDa}$, particularly with the Pan-Hsin raw water. Among three water samples, the TCI-HA sample had the highest DBPFP/DOC value and the Pan-Hsin raw water sample had the lowest DBPFP/DOC value in all ranges of molecular weight. The sequence of DBPFP/ DOC for three water samples is similar to that of $\mathrm{UV}_{254} /$ DOC shown in Fig. 1.

The distribution of DOC and DBPFP in three water samples at various molecular weight ranges is shown in Table 1. For Aldrich-HA and TCI-HA samples, DOC and DBPFP are distributed mostly in molecular weight greater than $30 \mathrm{kDa}$. As shown in Table 1, for the Aldrich-HA with molecular weight greater than $30 \mathrm{kDa}$, the distribution ratio of DOC is $46 \%$. In the same molecular weight range, the distribution ratios of 


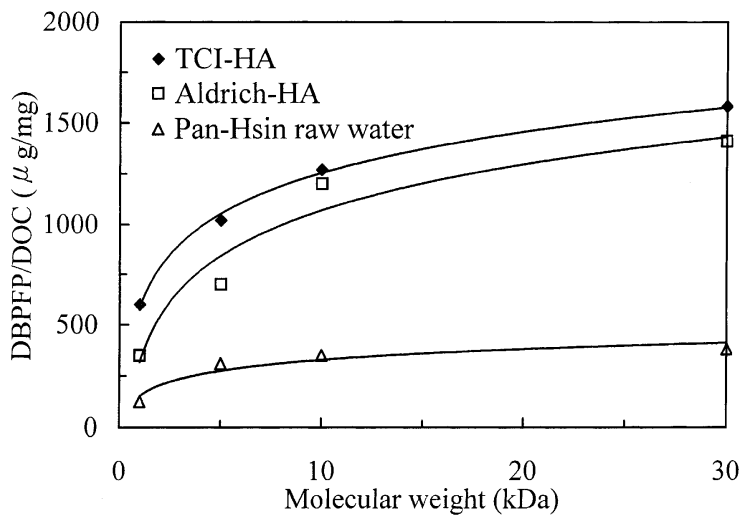

Fig. 3. The relationship between the total cumulated DBPFP/ DOC (the sum of DBP measured) concentration and the molecular weight.

$\mathrm{CHCl}_{3} \mathrm{FP}$, TCAAFP and DCAAFP are $53 \%, 51 \%$ and $39 \%$, respectively. For TCI-HA with molecular weight greater than $30 \mathrm{kDa}$, the distribution ratio of DOC is $51 \%$. The distribution ratios of $\mathrm{CHCl}_{3} \mathrm{FP}, \mathrm{TCAAFP}$ and DCAAFP average $40 \%(37-45 \%)$. The two humic acids contain high molecular weight organics which contribute to the majority of the DBPFP. However, the distribution of DBPFP per unit DOC is higher in the low molecular weight range (Figs. 2 and 3). In Table 1, the results of Pan-Hsin water samples suggest that DOC and DBPFP mainly come from low molecular weight organics $(<1 \mathrm{kDa})$, in which the distribution ratio of DOC and DBPFP $\left(\mathrm{CHCl}_{3} \mathrm{FP}\right.$, DCAAFP and TCAAFP $)$ are around $43 \%$ and $60 \%$, respectively.

\subsection{Chemical composition of DBP precursors in source water}

DOM isolated from the Pan-Hsin raw water was separated into four fractions. Fig. 4 shows the result of organic precursors in each fraction of the water sample. Most DOC is distributed in the hydrophilic and hydrophobic acid fractions, which account for $76 \%$ of DOC. As for DBPFP, the ability of THMFP per unit of DOC in each hydrophobic fraction is about two times that of the hydrophilic component. Moreover, the THM formation in the hydrophobic fraction decreases as the sequence of acids, bases and neutrals. On the other hand, the HAA generation in each fraction follows the order: hydrophobic acids $>$ hydrophobic neutrals $>$ hydrophobic bases $>$ hydrophilic material. Therefore, it is concluded that the hydrophobic acid organic matter possesses the highest potency of DBP formation; whereas the organic compounds mainly derives from both the hydrophilic and hydrophobic acid fractions.

Table 1

The distribution ratio of DOC and DBPFP among various molecular weight ranges in three waters*

\begin{tabular}{|c|c|c|c|c|c|c|c|c|c|c|}
\hline \multirow{2}{*}{$\begin{array}{l}\text { Range } \\
\text { (kDa) }\end{array}$} & \multirow[t]{2}{*}{ Parameter } & \multicolumn{3}{|c|}{ Aldrich-HA } & \multicolumn{3}{|l|}{ TCI-HA } & \multicolumn{3}{|c|}{ Pan-Hsin raw water } \\
\hline & & Average & S.D. & Range & Average & S.D. & Range & Average & S.D. & Range \\
\hline \multirow[t]{4}{*}{$<1$} & DOC & 20 & 1 & $20-21$ & 6 & 1 & $5-7$ & 43 & 3 & $40-46$ \\
\hline & $\mathrm{CHCl}_{3} \mathrm{FP}$ & 17 & 3 & $15-17$ & 16 & 2 & $15-20$ & 55 & 12 & $50-74$ \\
\hline & DCAAFP & 21 & 2 & $19-23$ & 15 & 2 & $13-17$ & 65 & 6 & $61-71$ \\
\hline & TCAAFP & 10 & 1 & $9-11$ & 18 & 1 & $18-20$ & 60 & 3 & $56-62$ \\
\hline \multirow[t]{4}{*}{$1-5$} & DOC & 5 & 4 & $2-9$ & 9 & 1 & $9-10$ & 12 & 4 & $9-16$ \\
\hline & $\mathrm{CHCl}_{3} \mathrm{FP}$ & 2 & 1 & $0-3$ & 19 & 3 & $16-22$ & 19 & 5 & $11-21$ \\
\hline & DCAAFP & 2 & 2 & $1-5$ & 17 & 5 & $14-23$ & 8 & 1 & $7-9$ \\
\hline & TCAAFP & 2 & 1 & $2-4$ & 16 & 1 & $15-17$ & 11 & 2 & $9-13$ \\
\hline \multirow[t]{4}{*}{$5-10$} & DOC & 7 & 2 & $5-7$ & 11 & 1 & $10-11$ & 14 & 9 & $8-24$ \\
\hline & $\mathrm{CHCl}_{3} \mathrm{FP}$ & 6 & 8 & $1-15$ & 12 & 3 & $10-15$ & 12 & 5 & $4-13$ \\
\hline & DCAAFP & 8 & 6 & $4-15$ & 10 & 3 & $8-13$ & 14 & 4 & $11-18$ \\
\hline & TCAAFP & 5 & 4 & $1-8$ & 13 & 1 & $12-14$ & 8 & 2 & $6-11$ \\
\hline \multirow[t]{4}{*}{$10-30$} & DOC & 20 & 4 & $17-24$ & 22 & 2 & $21-25$ & 14 & 4 & $11-18$ \\
\hline & $\mathrm{CHCl}_{3} \mathrm{FP}$ & 21 & 9 & $13-31$ & 7 & 2 & $6-9$ & 8 & 2 & $4-9$ \\
\hline & DCAAFP & 29 & 5 & $23-33$ & 10 & 4 & $7-14$ & 9 & 3 & $6-12$ \\
\hline & TCAAFP & 30 & 4 & $27-35$ & 10 & 2 & $8-12$ & 13 & 1 & $12-14$ \\
\hline \multirow[t]{4}{*}{$>30$} & DOC & 46 & 6 & $41-52$ & 51 & 2 & $50-53$ & 17 & 4 & $13-21$ \\
\hline & $\mathrm{CHCl}_{3} \mathrm{FP}$ & 53 & 8 & $47-63$ & 37 & 5 & $3-42$ & 5 & 5 & $5-14$ \\
\hline & DCAAFP & 39 & 11 & $29-51$ & 45 & 4 & $41-49$ & 8 & 1 & $7-9$ \\
\hline & TCAAFP & 51 & 8 & $46-49$ & 42 & 1 & $42-43$ & 8 & 3 & $5-11$ \\
\hline
\end{tabular}

${ }^{*}$ Data are all in $\%$. 


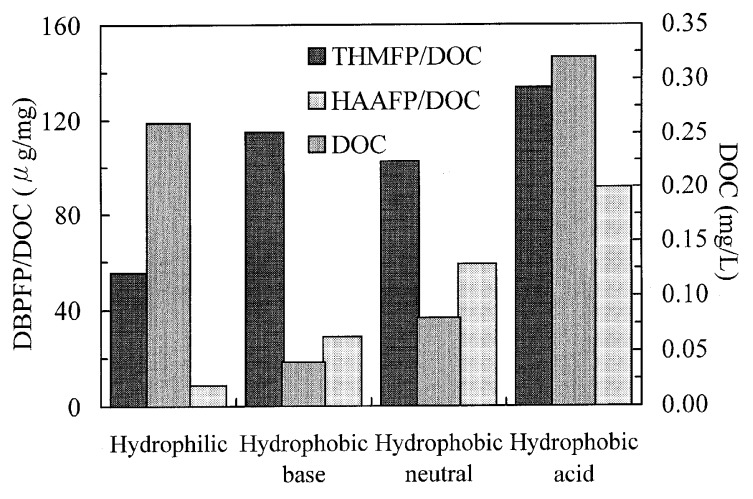

Fig. 4. The chemical composition of DBP precursors in PanHsin source water.

\subsection{Molecular weight distribution of DBP precursors in water purification processes}

Samples taken from each water treatment process at the Pan-Hsin Waterworks were used to evaluate the distribution pattern of molecular weight for DBP precursors. Table 2 shows the DOC concentration, distribution ratio and removal efficiency of each treatment process. In source water, $43 \%$ of the dissolved organic carbon has the molecular weight of $1 \mathrm{kDa}$ or less. On the other hand, the contribution from the other molecular weight ranges is comparable. It is observed that the removal efficiency of DOC increases proportionally to the molecular weight in the coagulation process, which has the highest DOC removal efficiency among all treatment processes. Effect of filtration on the removal of DOC is not significant. The total removal efficiency of DOC in the entire process also increases with the molecular weight. The larger precursor can be effectively removed due to its low polarity characteristics.

The $\mathrm{CHCl}_{3} \mathrm{FP}$ concentration, distribution ratio and removal efficiency of each treatment process are shown in Table 3. In source water, $55 \%$ of the $\mathrm{CHCl}_{3} \mathrm{FP}$ is contributed from the molecular weight of $1 \mathrm{kDa}$ or less, which is consistent with the results shown in Figs. 2 and 3. The trend in removing $\mathrm{CHCl}_{3} \mathrm{FP}$ by the coagulation process is similar to that of DOC as presented in Table 2. The removal efficiency of $\mathrm{CHCl}_{3} \mathrm{FP}$ in the coagulation process increases proportionally to the molecular weight. Although the coagulation process was effective in removing organic precursors or large molecule, it was not effective in eliminating small compounds.

Table 2

The DOC concentration, distribution ratio and removal efficiency for the effluent from each treatment process of Pan-Hsin Waterworks $^{\mathrm{a}}$

\begin{tabular}{|c|c|c|c|c|c|c|c|c|c|}
\hline \multirow{3}{*}{$\begin{array}{l}\text { Molecular } \\
\text { weight range } \\
(\mathrm{kDa})\end{array}$} & \multicolumn{9}{|c|}{ Treatment process } \\
\hline & \multicolumn{2}{|c|}{ Raw water } & \multicolumn{3}{|c|}{ Coagulation } & \multicolumn{3}{|c|}{ Filtration } & \multirow{2}{*}{$\frac{\text { Clear water }}{\text { IV }}$} \\
\hline & I & II & I & II & III & I & II & III & \\
\hline$<1$ & 0.34 & 43 & 0.31 & 51 & 9 & 0.25 & 47 & 18 & 31 \\
\hline $1-5$ & 0.09 & 12 & 0.07 & 12 & 21 & 0.07 & 13 & 4 & 26 \\
\hline $5-10$ & 0.11 & 14 & 0.08 & 13 & 28 & 0.07 & 14 & 6 & 50 \\
\hline $10-30$ & 0.11 & 14 & 0.07 & 12 & 34 & 0.07 & 13 & 5 & 57 \\
\hline$>30$ & 0.13 & 17 & 0.07 & 11 & 50 & 0.07 & 12 & 2 & 69 \\
\hline
\end{tabular}

${ }^{\mathrm{a}} \mathrm{I}$ : DOC concentration, $\mathrm{mg} / \mathrm{l}$; II: DOC distribution ratio, \%; III: DOC removal efficiency (compared to the previous unit process), $\%$; IV: DOC removal efficiency (compared to the raw water), \%.

Table 3

The $\mathrm{CHCl}_{3} \mathrm{FP}$ concentration, distribution ratio and removal efficiency for the effluent from each treatment process of Pan-Hsin Waterworks ${ }^{\mathrm{a}}$

\begin{tabular}{|c|c|c|c|c|c|c|c|c|c|}
\hline \multirow{3}{*}{$\begin{array}{l}\text { Molecular } \\
\text { weight range } \\
(\mathrm{kDa})\end{array}$} & \multicolumn{9}{|c|}{ Treatment process } \\
\hline & \multicolumn{2}{|c|}{ Raw water } & \multicolumn{3}{|c|}{ Coagulation } & \multicolumn{3}{|c|}{ Filtration } & \multirow{2}{*}{$\frac{\text { Clear water }}{\text { IV }}$} \\
\hline & I & II & I & II & III & I & II & III & \\
\hline$<1$ & 79 & 55 & 70 & 62 & 12 & 63 & 63 & 10 & 58 \\
\hline $1-5$ & 28 & 19 & 20 & 18 & 27 & 18 & 18 & 12 & 47 \\
\hline $5-10$ & 17 & 12 & 12 & 11 & 32 & 11 & 11 & 10 & 51 \\
\hline $10-30$ & 12 & 8 & 7 & 6 & 40 & 6 & 6 & 10 & 55 \\
\hline$>30$ & 8 & 5 & 3 & 3 & 64 & 2 & 2 & 15 & 69 \\
\hline
\end{tabular}

${ }^{\mathrm{a}} \mathrm{I}$ : $\mathrm{CHCl} 3 \mathrm{FP}$ concentration, $\mu \mathrm{g} / \mathrm{l}$; II: $\mathrm{CHCl} 3 \mathrm{FP}$ distribution ratio, \%; III: $\mathrm{CHCl} 3 \mathrm{FP}$ removal efficiency (compared to the previous unit process), \%; IV: CHCl3FP removal efficiency (compared to the raw water), $\%$. 


\section{Conclusions}

The $\mathrm{UV}_{254} / \mathrm{DOC}$ values of three water samples investigated in this study are in the following order: TCI$\mathrm{HA}>$ Aldrich-HA $>$ Pan-Hsin raw water. The $\mathrm{UV}_{254} /$ DOC values are inversely proportional to the molecular weight of the water samples. Since most of the DBPFPs are distributed in the low molecular weight range, it is concluded that small molecular precursors will play an important role in forming DBPs. Additionally, special attention should be drawn to the PanHsin Waterworks, because most of the DBP precursors in Pan-Hsin water samples are small organic compounds with the molecular weight less than $1 \mathrm{kDa}$. The hydrophobic acids have the greatest ability to form DBP, whereas the organic compounds mainly derive from both the hydrophilic and hydrophobic acid fractions. Therefore, effective removal of small molecule or hydrophobic acids prior to disinfection process will significantly reduce the DBP concentration in the finished water.

The coagulation removal efficiency of $\mathrm{CHCl}_{3} \mathrm{FP}$ and DOC increases proportionally to the molecular weight of the precursors. Although the coagulation process is effective in removing large organic precursors, it has limited efficiency in eliminating the small precursors, which possess the highest DBPFP. Since conventional coagulation process is ineffective in removing small organic molecules of $\mathrm{CHCl}_{3} \mathrm{FP}$, the enhanced coagulation technique should be provided to reduce the level of DBP concentrations in the finished waters.

\section{References}

APHA, 1995. Standard Methods for the Examination of Water and Wastewater, 19th Ed., American Public Health Association, Washington, DC.

Chang, C.N., 1999. Effect of molecular weight distribution of organic matter on the removal of disinfection by-products in the drinking water treatment plant. In: Proceedings of the Sixteenth Water Supply Conference in Taiwan, Taiwan, pp. 171-182 (in Chinese).

Collins, M.R., Amy, G.L., Steelink, C., 1986. Molecular weight distribution, carboxylic acidity and humic substance content of aquatic organic matter: implication for removal during water treatment. Environ. Sci. Technol. 20, 1024-1032.

Huang, W.J., Yeh, H.H., 1997. Effect of organic characteristics and bromide on disinfection by-products formation by chlorination. J. Environ. Sci. Health 32, 2311-2336.

Lou, J.C., Chiang, P.C., 1991. Study on mechanism of chlorination reaction of humic acids in drinking waters. J. Chinese Inst. Environ. Eng. 1, 57-64.

Owen, D.M., Amy, G.L., Chowdhury, Z.K., 1993. Characterization of Natural Organic Matter and its Relationship to Treatability. American Water Works Association Research Foundation, Denver, Colorado.

Veenstra, J.N., Schnoor, J.L., 1980. Seasonal variations in trihalomethane levels in an Iowa river water supply. J. Amer. Water Works Assoc. 72, 583-590. 